\title{
DETERMINATION OF THE COSY PROTON BEAM POLARIZATION USING THE WASA DETECTOR*
}

\author{
I. Schätti-Ozerianska, P. Moskal, M. Zieliński \\ the WASA-at-COSY Collaboration
}

The Marian Smoluchowski Institute of Physics, Jagiellonian University

Łojasiewicza 11, 30-348 Kraków, Poland

and

Institut für Kernphysik (IKP), Forschungszentrum Jülich

Wilhelm-Johnen-Straße, 52428 Jülich, Germany

(Received January 16, 2015)

The dynamics of $\eta$-meson production and the interaction of $\eta$ mesons with nucleons can be studied using the $\overrightarrow{p p} \rightarrow p p \eta$ reaction via measurements of the analyzing power $A_{y}$. To this end, we have performed a measurement of the $\overrightarrow{p p} \rightarrow p p \eta$ reaction using the WASA-at-COSY detector, which provides large acceptance and is $\varphi$-symmetric. The experiment was carried out for the beam momenta of $2026 \mathrm{MeV} / c$ and $2188 \mathrm{MeV} / c$. In this article, we present a method and results for the determination of the degree of the beam polarization.

DOI:10.5506/APhysPolB.46.153

PACS numbers: 13.88. + e, 24.70. + s

\section{Introduction}

Despite of a number of experiments [1-13] and theoretical approaches [14-22] still an open question remains about the mechanism of the $\eta$-meson production in nucleon-nucleon collisions. Previous studies concluded that the $\eta$-meson production in $p p$ and $p n$ reactions occurs predominantly via the $N^{*}(1535)$ resonance and that the proton $-\eta$ interaction is much larger than the interactions in the case of proton $-\pi^{0}$, and proton- $\eta^{\prime}$ [23-27]. For an unambiguous understanding of the $\eta$ production process, relative magnitudes from the partial waves contributions must be well established. The incompatibility between experiment and theory may be resolved by taking into account higher partial waves and additional baryon resonances [28]. This kind of study can be performed by measuring the analyzing power $A_{y}$ of the

* Funded by $\mathrm{SCOAP}^{3}$ under Creative Commons License, CC-BY 3.0. 
$\eta$ meson. Up to now, measurements of $A_{y}$ in the $\overrightarrow{p p} \rightarrow p p \eta$ reaction were conducted by the COSY-11 and DISTO collaborations [29-32]. However, these previous experiments suffered from low statistics and limited geometrical acceptance. In order to determine $A_{y}$ more accurately, the axially symmetric WASA detector [38] and the vertically polarized proton beam of COSY have been used to collect a high statistics data sample. An experiment was conducted for the beam momenta of $2026 \mathrm{MeV} / c$ and $2188 \mathrm{MeV} / c$ which correspond to excess energies of $15 \mathrm{MeV}$ and $72 \mathrm{MeV}$, respectively [35, 39]. The trigger was set to select events corresponding to the $\overrightarrow{p p} \rightarrow p p \eta$ and $\overrightarrow{p p} \rightarrow p p$ reactions. Proton-proton elastic scattering provides information for the determination of the polarization and the luminosity. Additionally, to control the effects caused by potential asymmetries in the detector setup the spin orientation was flipped from cycle to cycle.

\section{Extraction of the $\overrightarrow{p p} \rightarrow p p$ reaction}

The first step in the study of the asymmetry $A_{y}$ is the analysis of the reaction $\vec{p} p \rightarrow p p$. Based on the elastic scattering reaction, we have calculated the polarization degree for the whole data sample. In the $\overrightarrow{p p} \rightarrow p p$ reaction, one of the protons is registered in the Forward Detector, with geometrical acceptance of polar angle $\theta_{\mathrm{FD}}$ from $3^{\circ}$ to $18^{\circ}$ and the second is registered in the Central Detector. By utilizing $\Delta E-E$ method in the Forward Range Hodoscope and the angular correlation of the outgoing protons, we have selected events corresponding to the proton-proton elastic scattering. For further calculations, we have chosen only two $\theta_{\mathrm{FD}}^{*}$ ranges in the centre of mass: $\left[30^{\circ}-34^{\circ}\right]$ and $\left[34^{\circ}-38^{\circ}\right]$, for which $A_{y}$ is available from the EDDA database [41] and for which the number of events was sufficient. For precise background suppression, all events were divided into 36 bins in the azimuthal angle $\varphi$. Exemplary distribution of the $\theta_{\mathrm{FD}}^{*}$ vs. $\theta_{\mathrm{CD}}^{*}$ is shown in Fig. 1.

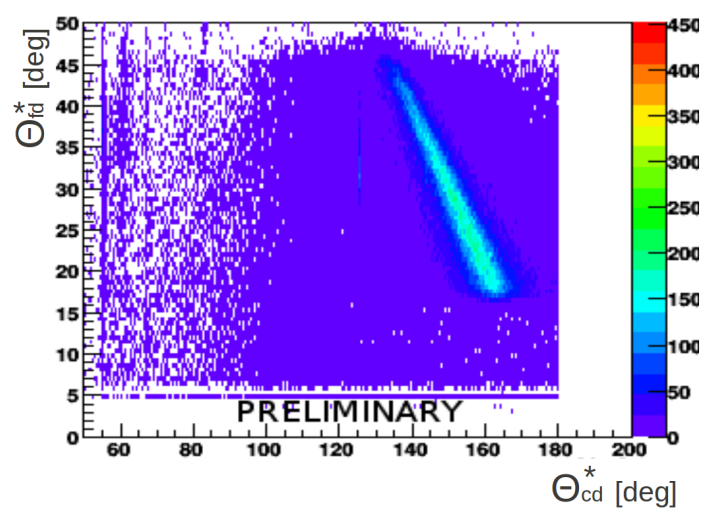

Fig. 1. Example of the $\theta_{\mathrm{FD}}^{*}$ vs. $\theta_{\mathrm{CD}}^{*}$ distribution. 
Figure 2 shows a clear asymmetry between angular distribution obtained for different spin orientations. In order to estimate the background, for each bin in $\theta_{\mathrm{FD}}^{*}$, the $\theta_{\mathrm{CD}}^{*}$ distribution was fitted separately with a fifth-order polynomial, excluding from the fit the range from $130^{\circ}$ to $160^{\circ}$, as shown in Fig. 3.
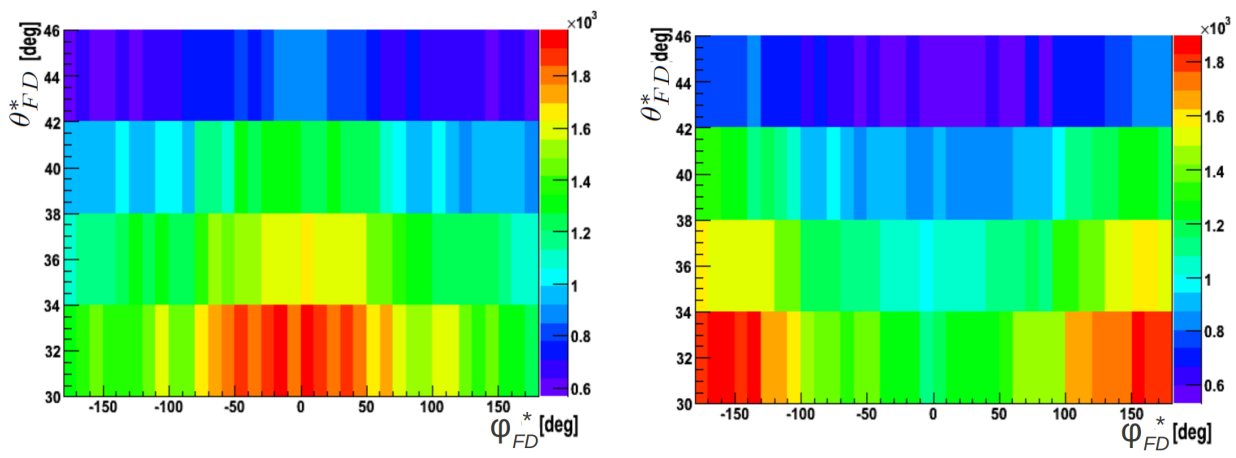

Fig. 2. Example of the angular distribution for the elastic scattered events with spin up polarization (left) and spin down (right).

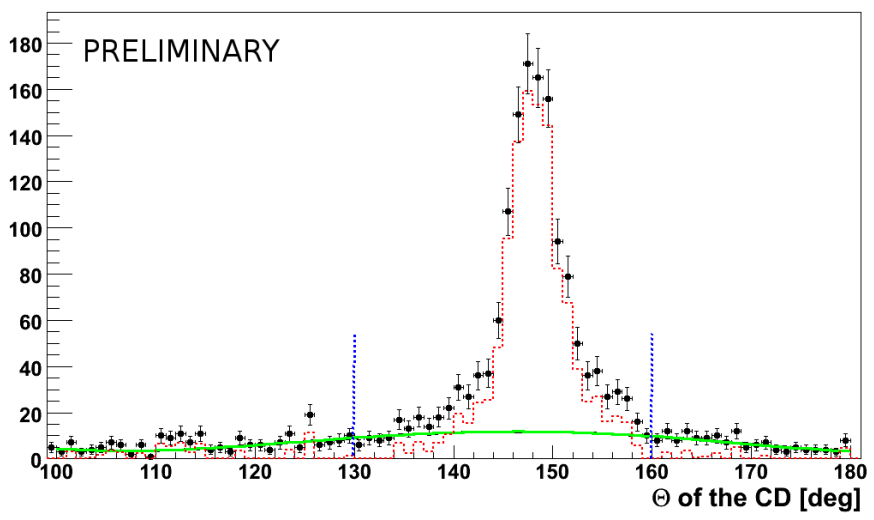

Fig. 3. The distribution of $\theta_{\mathrm{CD}}^{*}$ for $\theta_{\mathrm{FD}}^{*} \in\left[30^{\circ}, 34^{\circ}\right], \varphi \in\left[20^{\circ}, 30^{\circ}\right]$. Points denote data. Solid/green line corresponds to the fitted background, and dotted/red histogram shows the data after subtraction of the background.

\section{Polarization determination}

The polarization was determined from elastic scattering events by the following equation

$$
\frac{N^{\exp }(\theta, \varphi)-N^{\exp }(\theta, \varphi+180)}{N^{\exp }(\theta, \varphi)+N^{\exp }(\theta, \varphi+180)}=P \times \cos \varphi \times A_{y}(\theta)
$$


where $N^{\exp }(\theta, \varphi)$ and $N^{\exp }(\theta, \varphi+180)$ correspond to a given angular bin after correction for the acceptance, and $P$ denotes the polarization. $A_{y}(\theta)$ was calculated based on the results of the EDDA experiment [41]. The procedure is described in details elsewhere [36].

The polarization determined from all experimental data is shown in Fig. 4. The upper panel shows the results for measurements with a polarized beam obtained for both studied beam momenta. The lower panel presents the polarization of the control sample, which has been measured with an unpolarized beam. In the case of the control sample, the measured polarization is consistent with zero, within the statistical uncertainty. Figure 4 shows the results before and after the correction for shifts of the center of the beam-target interaction region from its nominal position $[36,37]$.
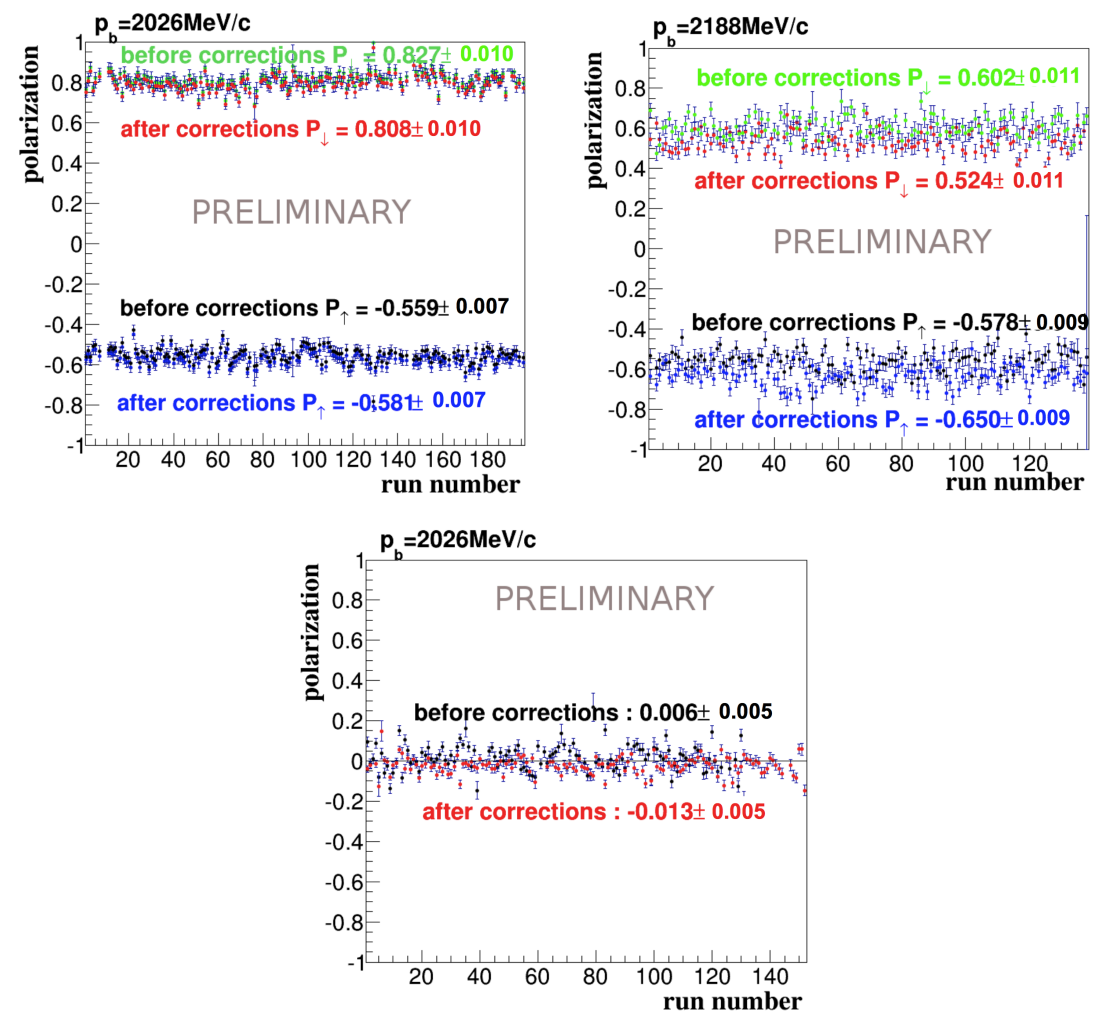

Fig. 4. Distributions of polarization as a function of run number for polarized (upper panel) and unpolarized (lower panel) data. Results for both polarization modes are shown.

The average polarization of all acquired data was calculated analyzing all events together. The results are shown in Table I. 
TABLE I

Preliminary polarization achieved in the WASA-at-COSY experiment conducted in the year 2010 .

\begin{tabular}{ccr}
\hline \hline$p_{b}[\mathrm{GeV} / c]$ & Spin mode & \multicolumn{1}{c}{ Polarization } \\
\hline 2026 & down & $0.793 \pm 0.010$ \\
& up & $-0.577 \pm 0.007$ \\
\hline 2188 & down & $0.537 \pm 0.009$ \\
& up & $-0.635 \pm 0.011$ \\
\hline 2026 & unpolarized & $-0.012 \pm 0.005$
\end{tabular}

\section{Summary}

The polarization of elastic scattering events in the reaction $\overrightarrow{p p} \rightarrow p p$ has been presented. The polarization differs between the spin modes. However, it is stable between runs for the whole period of data taking. Additionally, the value of the polarization for an unpolarized beam has been measured to be consistent with zero, as expected. A high degree of polarization for both beam momenta gives a very good perspective for the determination of the analyzing power for the $\overrightarrow{p p} \rightarrow p p \eta$ reaction.

We acknowledge support by the Polish National Science Center through grant No. 2011/03/B/ST2/01847, and by the FFE grants of the Research Center Jülich.

\section{REFERENCES}

[1] E. Chiavassa et al., Phys. Lett. B322, 270 (1994).

[2] H. Calén et al., Phys. Lett. B366, 39 (1996).

[3] H. Calén et al., Phys. Rev. Lett. 79, 2642 (1997).

[4] F. Hibou et al., Phys. Lett. B438, 41 (1998).

[5] J. Smyrski et al., Phys. Lett. B474, 182 (2000).

[6] A.M. Bergdolt et al., Phys. Rev. D48, 2969 (1993).

[7] M. Abdel-Bary et al., Eur. Phys. J. A16, 127 (2003).

[8] H. Calén et al., Phys. Rev. C58, 2667 (1998).

[9] P. Moskal et al., Phys. Rev. C69, 025203 (2004).

[10] P. Moskal et al., Eur. Phys. J. A43, 131 (2010).

[11] H. Petren et al., Phys. Rev. C82, 055206 (2010).

[12] H. Calen et al., Phys. Rev. Lett. 79, 2642 (1997). 
[13] P. Moskal et al., Phys. Rev. C79, 015208 (2009).

[14] C. Wilkin, Acta Phys. Pol. B 41, 2191 (2010).

[15] K. Nakayama et al., Phys. Rev. C65, 045210 (2002).

[16] G. Fäldt, C. Wilkin, Phys. Scr. 64, 427 (2001).

[17] J.F. Germond et al., Nucl. Phys. A518, 308 (1990).

[18] J.M. Laget et al., Phys. Lett. B257, 254 (1991).

[19] A. Moalem et al., Nucl. Phys. A600, 445 (1996).

[20] T. Vetter et al., Phys. Lett. B263, 153 (1991).

[21] B.L. Alvaredo et al., Phys. Lett. B324, 125 (1994).

[22] M. Batini c et al., Phys. Scr. 56, 321 (1997).

[23] P. Moskal et al., Phys. Lett. B482, 356 (2000).

[24] P. Moskal et al., Prog. Part. Nucl. Phys. 49, 1 (2002).

[25] E. Czerwinski et al., Phys. Rev. Lett. 113, 062004 (2014).

[26] E. Czerwinski et al., Acta Phys. Pol. B 45, 739 (2014).

[27] M. Nanova et al., Phys. Lett. B727, 417 (2013).

[28] K. Nakayama et al., Phys. Rev. C68, 045201 (2003).

[29] R. Czyżykiewicz et al., Phys. Rev. Lett. 98, 122003 (2007).

[30] P. Winter et al., Eur. Phys. J. A18, 355 (2003).

[31] P. Winter et al., Phys. Lett. B544, 251 (2002) [Erratum ibid., B553, 339 (2003)].

[32] F. Balestra et al., Phys. Rev. C69, 064003 (2004).

[33] P. Klaja et al., AIP Conf. Proc. 796, 160 (2005).

[34] S. Brauksiepe et al., Nucl. Instrum. Methods Phys. Res. A376, 397 (1996).

[35] P. Moskal, M. Hodana, J. Phys. Conf. Ser. 295, 012080 (2011);

[arXiv:1101.5486 [nucl-ex]].

[36] M. Hodana et al., Acta Phys. Pol. B Proc. Suppl. 6, 1041 (2013).

[37] M. Hodana et al., Acta Phys. Pol. B 45, 697 (2014).

[38] H.H. Adam et al., arXiv:nucl-ex/0411038.

[39] P. Moskal, M. Hodana, H. Calén, Proposal to COSY-PAC, 185.1, 2010.

[40] L. Demirors, Ph.D. Thesis, Hamburg University, 2005.

[41] M. Altmeier et al., Phys. Rev. Lett. 85, 1819 (2000). 